\title{
The Parameterization Reduction of Soft Point and its Applications with Soft Matrix
}

\author{
Güzide Şenel \\ Department of Elementary Education, \\ Amasya University, 05100 Amasya, Turkey
}

\begin{abstract}
The innovation about soft point in this study is, soft point's soft matrix form which were not described before is defined for each set of parameters. The matrix representation of soft points is useful for storing all soft points that can be obtained in all different parameters. The proposed soft matrix provides every soft point that changes with each parameter that takes place in a soft set is proved and showed that it enables detailed examination in application of soft set theory.
\end{abstract}

\section{General Terms}

2010 AMS Classification: $03 G 25,20 D 05$

\section{Keywords}

Soft set, soft point, soft matrix, soft matrix form of soft point.

\section{INTRODUCTION}

Classical method to solve complicated problem in economics, engineering and environment because of various uncertainties typical for those problems can not be successfully used. There are theories, viz. theory of probability, theory of fuzzy sets, theory of vague set [16], theory of interval mathematics [23] and theory of rough sets [24] which can be consider as mathematical tools for dealing with uncertainties. But all these theories have their own difficulties. The reason of the difficulties is, possibly, the inadequacy of the parametrization tool of the theories. To overcome these difficulties Molodtsov [19] introduced the concept of soft set as a new mathematical tool for dealing with uncertainties which is free from the difficulties that have troubled the usual theoretical approaches. The parametrization tool of soft set theory enhance the flexibility of its applications. Maji et al. [17, 18] introduced several applications of soft sets in decision making problems. There has been some progress concerning practical applications of soft set theory, especially the use of soft sets in decision making. So, the properties and applications of soft set theory have been studied increasingly [1, 2, 4, 5, 6, 7, 15, 18, 19, 20, 21].

Matrices play an important role in the broad area of science and engineering. However, the classical matrix theory sometimes fails to solve the problems involving uncertainties, occurring in an imprecise environment. In [5], initiated a matrix representation of a soft set and successfully applied the proposed notion of soft ma- trix in certain decision making problems. Also the soft max - min decision making method was set up in it. The very common operations of soft matrices are defined in [22] like AND, OR, union, intersection, commutative, associative, distributive, De Morgan's laws and convergent property for soft matrices are investigated. In [27] Thomason, introduced the fuzzy matrices to represent fuzzy relation in a system based on fuzzy set theory and discussed about the convergence of powers of fuzzy matrix. In [12, 13 14], some important results on determinant of a square fuzzy matrices are discussed. Thereafter, in [10|, T.J. Neog, D. K. Sutwere extended this new concept to soft set theory, introducing a new concept called fuzzy soft set based on fuzzy reference function. Recently, N. TridivJyoti et al. [11] combined fuzzy soft set based on reference function with soft matrices.

The concept of point generalized to the theory of soft set in [3]. According to the definition of soft point, Zorlutuna et al.|28] introduce constant soft mapping and observe that the image of a soft set under constant soft mapping is a soft point as in classical set theory. S. Das et al.[9] introduced the concept of soft point and using this concept they have given some new definitions in soft set theory. In this study, all definitions were examined for soft point defined in [3, 9, 28]. According to the definitions, being a soft point the parameters in the set $X$ must have a response of one and only one in the initial universe. Therefore, the provision of such parameters do not need to show on the symbol of soft point with a fixed set of parameters, has to show by indexed parameters. We know that $f$ soft set's all soft points specify one and each soft set. More than one soft point can be generated with a parameter $e$. The innovation about soft point in this study is, soft point's soft matrix form which is useful for storing all soft points that can be obtained in all different parameters is defined.

Since matrix is a very important tool in any branches of mathematics mentioned above so we motivated to study it over soft points and provide better understanding the concept of soft points with soft matrix.

In this study, the concept of soft point is analized by generating a useful method with soft matrix. Different applications of soft matrix of a soft point that were not described before is presented. Case studies of current practice are presented to course development and delivery of soft set theory. The paper unfolds as follows. In the next section, we briefly introduce some definitions related to soft set and soft real set. In section 3, the definition of soft point for each set of parameters is presented. Also, the notion of soft matrix of 
soft points is given and its applications are presented. Conclusion is appear in the last section.

\section{PRELIMINARY}

In this section, some basic notions of soft set theory which may be found in $[6,7,15,18,19,25,28]$ are recalled for further details. Throughout this work, $U$ refers to an initial universe, $E$ is a set of parameters, $A \subseteq E$ and $P(U)$ is the power set of $U$.

DEFINITION 1. ([6],[19]) A soft set $f_{A}$ on the universe $U$ is a set defined by

$$
f_{A}: E \rightarrow \mathcal{P}(U) \text { suchthat } f_{A}(x)=\emptyset \text { if } \quad x \notin A .
$$

Here $f_{A}$ is also called an approximate function. A soft set over $U$ can be represented by the set of ordered pairs

$$
f_{A}=\left\{\left(x, f_{A}(x)\right): x \in E, f_{A}(x) \in P(U)\right\} .
$$

Note that the set of all soft sets over $U$ will be denoted by $S$.

DEFINITION 2. ([6]) Let $f_{A} \in S$. Then,

If $f_{A}(x)=\emptyset$ for all $x \in E$, then $f_{A}$ is called an empty set, denoted by $\Phi$.

If $f_{A}(x)=U$ for all $x \in E$, then $f_{A}$ is called universal soft set, denoted by $\tilde{E}$.

If $f_{A}(x)$ is a singleton set for all $x \in E$, then $f_{A}$ is called a singleton soft set.

Definition 3. ([6]) Let $f_{A}, f_{B} \in S$. Then,

$f_{A}$ is a soft subset of $f_{B}$, denoted by $f_{A} \widetilde{\subseteq} f_{B}$, if $f_{A}(x) \subseteq f_{B}(x)$ for all $x \in E$.

Definition 4. ([6]) Let $f_{A}, f_{B} \in S$. Then, union of $f_{A}$ and $f_{B}$, denoted by $f_{A} \tilde{\cup} f_{B}$, is defined as $f_{A} \tilde{\cup} f_{B}=f_{A \tilde{\cup} B}$, where $f_{A \cup \tilde{B}}=f_{A}(x) \cup f_{B}(x)$ for all $x \in E$.

DEFINITION 5. ([6]) Let $f_{A}, f_{B} \in S$. Then, intersection of $f_{A}$ and $f_{B}$, denoted by $f_{A} \tilde{\cap} f_{B}$, is defined as $f_{A} \tilde{\cap} f_{B}=f_{A \tilde{\cap} B}$, where $f_{A \tilde{\cap} B}=f_{A}(x) \cap f_{B}(x)$ for all $x \in E$.

DEFINITION 6. ([87) Let $X$ be a non-empty set and $E$ be a non-empty parameter set. Then a function $\varepsilon: E \rightarrow X$ is said to be a soft element of $X$. A soft element $\varepsilon$ of $X$ is said to belong to a soft set $A$ of $X$, denoted by $\varepsilon \in A$, if $\varepsilon(e) \in A(e), \forall e \in E$. Thus a soft set $A$ of $X$ with respect to the index set $E$ can be expressed as $A(e)=\{\varepsilon(e), \varepsilon \in A\}, e \in E$.

DEFINITION 7. ([8]) Let $R$ be the set of real numbers, $\mathcal{P}(R)$ be the collection of all non-empty bounded subsets of $R, E$ be a set of parameters and $A \subseteq E$. Then a mapping $f: A \rightarrow \mathcal{P}(R)$ is called a soft real set. It is denoted by $(f, A)$. If in particular $(f, A)$ is a singleton soft set, then identifying $(f, A)$ with the corresponding soft element, it will be called a soft real number.

From now on, the single parameter set to be used is denoted by $E$ to define soft sets and their operations. Thus, the subscript $E$ can be deleted from the soft sets $(f, E)$, i.e, a soft set $f_{E}$ will be denoted shortly by $f$ unless this will cause confusion. In general, soft sets and their approximate functions are denoted by $f, g, h, \ldots$, and soft sets and their approximate functions are used interchangeably.

DEFINITION 8. Let us denote the collection of all soft points of $f$ by $S P(f)$, the set of all soft real sets by $R(E)$, the set of all soft real numbers by $\tilde{R}(E)$ and the set of all non-negative soft real numbers by $\tilde{R}^{*}(E)$. If a soft real set is a singleton soft set, it will be called a soft real number and denoted by $\tilde{r}, \tilde{s}$ etc.

\section{SOFT POINT}

The innovation about soft point in this section is, soft point's soft matrix form based on [3] which is representative of the soft sets is defined. This style of representation is useful for storing all soft points that can be obtained in all different parameters.

DEFINITION 9. ([3]) The soft set $f$ is called a soft point in $S$, if for the parameter $e_{i} \in E$ such that $f\left(e_{i}\right) \neq \emptyset$ and $f\left(e_{j}\right)=\emptyset$, for all $e_{j} \in E \backslash\left\{e_{i}\right\}$ is denoted by $\left(e_{i_{f}}\right)_{j}$ for all $i, j \in N^{+}$.

Note that the set of all soft points of $f$ will be denoted by $S P(f)$.

Let $U=\left\{u_{1}, u_{2}, u_{3}, u_{4}, u_{5}\right\}$ and $E=\left\{e_{1}, e_{2}, e_{3}\right\} . f \in S$ and

$$
f=\left\{\left(e_{1},\left\{u_{1}, u_{3}\right\}\right),\left(e_{2},\left\{u_{2}, u_{3}\right\}\right),\left(e_{3},\left\{u_{1}, u_{2}, u_{3}\right\}\right)\right\}
$$

Then the soft points for the parameter $e_{1}$ are;

$$
\begin{aligned}
& \left(e_{1_{f}}\right)_{1}=\left(e_{1},\left\{u_{1}\right\}\right) \\
& \left(e_{1_{f}}\right)_{2}=\left(e_{1},\left\{u_{3}\right\}\right) \\
& \left(e_{1_{f}}\right)_{3}=\left(e_{1},\left\{u_{1}, u_{3}\right\}\right)
\end{aligned}
$$

one of them can be chosen as soft point.

For the the parameter $e_{2}$ one of three occasions can be chosen as soft point likewise;

$$
\begin{aligned}
& \left(e_{2_{f}}\right)_{1}=\left(e_{2},\left\{u_{2}\right\}\right) \\
& \left(e_{2_{f}}\right)_{2}=\left(e_{2},\left\{u_{3}\right\}\right) \\
& \left(e_{2_{f}}\right)_{3}=\left(e_{2},\left\{u_{2}, u_{3}\right\}\right)
\end{aligned}
$$

The soft points for the parameter $e_{3}$ are;

$$
\begin{aligned}
& \left(e_{3_{f}}\right)_{1}=\left(e_{3},\left\{u_{1}\right\}\right) \\
& \left(e_{3_{f}}\right)_{2}=\left(e_{3},\left\{u_{2}\right\}\right) \\
& \left(e_{3_{f}}\right)_{3}=\left(e_{3},\left\{u_{3}\right\}\right) \\
& \left(e_{3_{f}}\right)_{4}=\left(e_{3},\left\{u_{1}, u_{2}\right\}\right) \\
& \left(e_{3_{f}}\right)_{5}=\left(e_{3},\left\{u_{1}, u_{3}\right\}\right) \\
& \left(e_{3_{f}}\right)_{6}=\left(e_{3},\left\{u_{2}, u_{3}\right\}\right) \\
& \left(e_{3_{f}}\right)_{7}=\left(e_{3},\left\{u_{1}, u_{2}, u_{3}\right\}\right)
\end{aligned}
$$

DEFINITION 10. ([26]) Let $f \in S$. Then, If $f(e)=\emptyset$ for all $e \in E$, then $f$ is called null soft point, denoted by $e_{\Phi}$.

If $f(e)=U$ for all $e \in E$, then $f$ is called universal soft point, denoted by $e_{\tilde{E}}$.

If there is only one parameter $e \in E$ in $f$, then $f$ is denoted by $e_{f_{i}}$. If there is only one parameter $e \in E$ in $f$, and $f(e)=\{u\}$ then $f$ is denoted by $e_{f}$.

DEFINITION 11. ([28]) The soft point $\left(e_{i_{f}}\right)_{j}$ is said to belong to a soft set $g \in S$, denoted by $\left(e_{i_{f}}\right)_{j} \tilde{\in} g$, if for the parameter $e_{i} \in E$ and $f\left(e_{i}\right) \subseteq g\left(e_{i}\right)$.

([28]) Let $E$ and $U$ be finite sets. All the soft points of soft set $f$ is equal to

$$
\sum_{e \in E}\left(2^{|f(e)|}-1\right)
$$

where $|f(e)|$ is cardinality of $f\left(e_{i}\right)$.

DEFINITION 12. ([28]) The soft points $\left(e_{i_{f}}\right)_{j}$ and $\left(e_{k_{f}}\right)_{s}$ are said to be equal if $e_{i}=e_{k}$ and $f\left(e_{i}\right)=f\left(e_{k}\right)$. 
It is clearly from the definition of soft point that if $e_{i} \neq e_{k}$ then, $\left(e_{i_{f}}\right)_{j} \neq\left(e_{k_{f}}\right)_{s}$. But being $\left(e_{i_{f}}\right)_{j} \neq\left(e_{k_{f}}\right)_{s}$ is not implies to be $e_{i} \neq e_{k}$ as seen in the following example: Consider the soft set $f$ is defined in Example 3. soft points $\left(e_{1_{f}}\right)_{1}=\left(e_{1},\left\{u_{1}\right\}\right)$ and $\left(e_{1_{f}}\right)_{2}=\left(e_{1},\left\{u_{3}\right\}\right)$ are written by the parameter $e_{1}$ but they are different. ([9]) The union of any collection of soft points can be considered as a soft set and every soft set can be expressed as union of all soft points belonging to it. It can be shown as,

$$
f=\tilde{U}\left(e_{i_{f}}\right)_{j} \tilde{\epsilon} f\left(e_{i_{f}}\right)_{j}
$$

Let $f=\left\{\left(e_{1},\left\{u_{2}\right\}\right),\left(e_{2},\left\{u_{1}, u_{2}, u_{3}\right\}\right)\right\}$. All the soft points of $f$ are;

$$
\begin{aligned}
& \left\{\left(e_{1_{f}}\right)_{1}\right\}=\left\{\left(e_{1},\left\{u_{2}\right\}\right)\right\} \\
& \left\{\left(e_{2_{f}}\right)_{1}\right\}=\left\{\left(e_{2},\left\{u_{1}\right\}\right)\right\} \\
& \left\{\left(e_{2_{f}}\right)_{2}\right\}=\left\{\left(e_{2},\left\{u_{2}\right\}\right)\right\} \\
& \left\{\left(e_{2_{f}}\right)_{3}\right\}=\left\{\left(e_{2},\left\{u_{3}\right\}\right)\right\} \\
& \left\{\left(e_{2_{f}}\right)_{4}\right\}=\left\{\left(e_{2},\left\{u_{1}, u_{2}, u_{3}\right\}\right)\right\}
\end{aligned}
$$

Here we get

$$
f=\sim_{\bigcup^{2}}^{2}\left(\tilde{U}^{3=1}\left(\left\{e_{i_{f}}\right\}\right)_{j}\right)
$$

([28]) Let $f, g \tilde{\in} S$. Then,

[i.] $f \widetilde{\subseteq} g$, if $\left(e_{i_{f}}\right)_{j} \tilde{\in} f$ then $\left(e_{i_{f}}\right)_{j} \tilde{\in} g$ for all $e_{i} \tilde{\in} f . f=g$, if and only if $\left(e_{i_{f}}\right)_{j} \tilde{\in} f$ then $\left(e_{i_{f}}\right)_{j} \tilde{\in} g$ and $\left(e_{i_{f}}\right)_{j} \tilde{\in} g$ and $\left(e_{i_{f}}\right)_{j} \tilde{\in} f$ for all $e_{i} \tilde{\in} f$.

Let $f \tilde{\in} S$. If $\left(e_{i_{f}}\right)_{j} \tilde{\in} f$ then, $\left(e_{i_{f}}\right)_{j} \notin \tilde{f} f^{\tilde{c}} . \quad\left(e_{i_{f}}\right)_{j} \tilde{\in} f \tilde{\cup} g$ does not imply that $\left(e_{i_{f}}\right)_{j} \tilde{\in} f$ or $\left(e_{i_{f}}\right)_{j} \tilde{\in} g$. It is shown in the following example: Let $U=\left\{u_{1}, u_{2}, u_{3}\right\}$ and $E=\left\{e_{1}, e_{2}, e_{3}\right\}$. $f, g \tilde{\in} S$ and

$$
f=\left\{\left(e_{1},\left\{u_{1}\right\}\right),\left(e_{2},\left\{u_{1}, u_{3}\right\}\right),\left(e_{3},\left\{u_{1}, u_{2}, u_{3}\right\}\right)\right\}
$$

and

$$
g=\left\{\left(e_{1},\left\{u_{2}, u_{3}\right\}\right),\left(e_{2},\left\{u_{1}, u_{2}\right\}\right),\left(e_{3},\left\{u_{2}\right\}\right)\right\}
$$

Hence, $f \tilde{\cup} g=\left\{\left(e_{1}, U\right),\left(e_{2}, U\right),\left(e_{3}, U\right)\right\}$ and $e_{1_{E}} \tilde{\in} f \tilde{\cup} g$. But $e_{1_{E}} \notin \tilde{f}$ and $e_{1_{E}} \notin \tilde{g} g$.

\subsection{Soft matrix of soft point}

More than one soft point can be generated with a parameter $e$. Let $e_{i}, i \in N^{+}$, be these soft points, having soft set $f_{j}, j \in N^{+}$, equivalent to each parameter $e_{i},\left(e_{i_{f}}\right)_{j}$ soft point can be defined by a soft matrix. According to this, definition of soft point is redesingned by soft matrix as taking into account all soft points that can be obtained in all different parameters.

(2) Definition 13. Let $E=\left\{e_{1}, e_{2}, \ldots\right\}, f \tilde{\in} S$ and $\left(e_{i_{f}}\right)_{j} \tilde{\epsilon} f$. Then the soft point $\left(e_{i_{f}}\right)_{j}$ can be presented by a soft matrix as in the following form:

\begin{tabular}{c|ccccccc}
$\left(e_{i_{f}}\right)_{j}$ & $f\left(e_{1}\right)$ & $f\left(e_{2}\right)$ & $\cdots$ & $f\left(e_{j}\right)$ & $\cdots$ & $f\left(e_{i}\right)$ & $\cdots$ \\
\hline$f_{1}\left(e_{i}\right)$ & $a_{11}$ & $a_{12}$ & $\cdots$ & $a_{1 j}$ & $\cdots$ & $a_{1 i}$ & $\cdots$ \\
$f_{2}\left(e_{i}\right)$ & $a_{21}$ & $a_{22}$ & $\cdots$ & $a_{2 j}$ & $\cdots$ & $a_{2 i}$ & $\cdots$ \\
$\vdots$ & $\vdots$ & $\vdots$ & & $\vdots$ & & $\vdots$ & \\
$f_{r}\left(e_{i}\right)$ & $a_{r 1}$ & $a_{r 2}$ & $\cdots$ & $a_{r j}$ & $\cdots$ & $a_{r i}$ & $\cdots$ \\
$\vdots$ & $\vdots$ & $\vdots$ & & $\vdots$ & & $\vdots$ &
\end{tabular}

where

$a_{k \ell}=\left\{\sim r, f_{k}\left(e_{\ell}\right) \in f\left(e_{\ell}\right), \tilde{r} \tilde{\in} \tilde{R}(E), \tilde{0}, f_{k}\left(e_{\ell}\right) \notin f\left(e_{\ell}\right), \tilde{0} \tilde{\in} \tilde{R}(E)\right.$.

\subsection{Application}

From now on to the next section, the definition of the soft matrix form of the soft point $\left(e_{i_{f}}\right)_{j}$ which is defined in Definition 13 will be used. To illustrate matrix representation of soft point the following example is considered: Let $U=\left\{u_{1}, u_{2}, u_{3}\right\}$ and $E=\left\{e_{1}, e_{2}\right\} . f \in S$ and

$$
f=\left\{\left(e_{1},\left\{u_{1}\right\}\right),\left(e_{2},\left\{u_{2}, u_{3}\right\}\right)\right\}
$$

Then,

$$
\begin{aligned}
& f_{1}=\left\{\left(e_{1},\left\{u_{1}\right\}\right)\right\}, \\
& f_{2}=\left\{\left(e_{2},\left\{u_{2}\right\}\right)\right\}, \\
& f_{3}=\left\{\left(e_{2},\left\{u_{3}\right\}\right)\right\}, \\
& f_{4}=\left\{\left(e_{2},\left\{u_{2}, u_{3}\right\}\right)\right\}, \\
& f_{5}=\left\{\left(e_{1},\left\{u_{1}\right\}\right),\left(e_{2},\left\{u_{2}\right\}\right)\right\}, \\
& f_{6}=\left\{\left(e_{1},\left\{u_{1}\right\}\right),\left(e_{2},\left\{u_{3}\right\}\right)\right\}, \\
& f_{7}=f, \\
& f_{8}=\Phi
\end{aligned}
$$

Then the soft point for the parameter $e_{1}$ is;

$$
\left(e_{1_{f}}\right)_{1}=\left(e_{1},\left\{u_{1}\right\}\right)
$$

one of them can be chosen as soft point.

For the the parameter $e_{2}$ one of three occasions can be chosen as soft point;

$$
\begin{aligned}
& \left(e_{2_{f}}\right)_{1}=\left(e_{2},\left\{u_{2}\right\}\right) \\
& \left(e_{2_{f}}\right)_{2}=\left(e_{2},\left\{u_{3}\right\}\right) \\
& \left(e_{2_{f}}\right)_{3}=\left(e_{2},\left\{u_{2}, u_{3}\right\}\right)
\end{aligned}
$$

Then the matrix represantations of soft points are;

$$
\begin{array}{c|c}
\left(e_{1_{f}}\right)_{j} & f\left(e_{1}\right) \\
\hline f_{1}\left(e_{1}\right) & a_{11}=\tilde{r} \\
f_{2}\left(e_{1}\right) & a_{21}=\tilde{0} \\
f_{3}\left(e_{1}\right) & a_{31}=\tilde{0} \\
f_{4}\left(e_{1}\right) & a_{41}=\tilde{0} \\
f_{5}\left(e_{1}\right) & a_{51}=\tilde{r} \\
f_{6}\left(e_{1}\right) & a_{61}=\tilde{r} \\
f_{7}\left(e_{1}\right) & a_{71}=\tilde{r} \\
f_{8}\left(e_{1}\right) & a_{81}=\tilde{0}
\end{array}
$$


and

\begin{tabular}{c|c}
$\left(e_{2_{f}}\right)_{j}$ & $f\left(e_{2}\right)$ \\
\hline$f_{1}\left(e_{2}\right)$ & $a_{12}=\tilde{0}$ \\
$f_{2}\left(e_{2}\right)$ & $a_{22}=\tilde{r}$ \\
$f_{3}\left(e_{2}\right)$ & $a_{32}=\tilde{r}$ \\
$f_{4}\left(e_{2}\right)$ & $a_{42}=\tilde{r}$ \\
$f_{5}\left(e_{2}\right)$ & $a_{52}=\tilde{r}$ \\
$f_{6}\left(e_{2}\right)$ & $a_{62}=\tilde{r}$ \\
$f_{7}\left(e_{2}\right)$ & $a_{72}=\tilde{r}$ \\
$f_{8}\left(e_{2}\right)$ & $a_{82}=\tilde{0}$
\end{tabular}

All the soft points can be shown by the following soft matrix:

\begin{tabular}{c|cc}
$\left(e_{i_{f}}\right)_{j}$ & $f\left(e_{1}\right)$ & $f\left(e_{2}\right)$ \\
\hline$f_{1}\left(e_{i}\right)$ & $\tilde{r}$ & $\tilde{0}$ \\
$f_{2}\left(e_{i}\right)$ & $\tilde{0}$ & $\tilde{r}$ \\
$f_{3}\left(e_{i}\right)$ & $\tilde{0}$ & $\tilde{r}$ \\
$f_{4}\left(e_{i}\right)$ & $\tilde{0}$ & $\tilde{r}$ \\
$f_{5}\left(e_{i}\right)$ & $\tilde{r}$ & $\tilde{r}$ \\
$f_{6}\left(e_{i}\right)$ & $\tilde{r}$ & $\tilde{r}$ \\
$f_{7}\left(e_{i}\right)$ & $\tilde{r}$ & $\tilde{r}$ \\
$f_{8}\left(e_{i}\right)$ & $\tilde{0}$ & $\tilde{0}$
\end{tabular}

The following propositions were accepted as a generalization of classical set theory in [25]. Now, we study them with soft real numbers in dept and give their proofs using soft matrix form of soft points. From now on, we will use arithmetic operations on soft real sets based on study of $[8]$.

$$
|\tilde{r}+\tilde{s}|=|\tilde{r}|+|\tilde{s}|
$$

for all $\tilde{r}, \tilde{s} \in \tilde{R}(E)$. 1st Case : Let $f_{k}\left(e_{\ell}\right) \in f\left(e_{\ell}\right)$ for all $\ell \in N^{+}$. Then, from the Definition[13

$$
\tilde{r}=a_{k i} \text { and } \quad \tilde{s}=a_{k j}
$$

for all $k \in N$. So that,

$$
|\tilde{r}+\tilde{s}|=\left|a_{k i}+a_{k j}\right|
$$

On the other hand, since

$$
|\tilde{r}|+|\tilde{s}|=\left|a_{k i}\right|+\left|a_{k j}\right|
$$

As we know that $\left|a_{k i}+a_{k j}\right|=\left|a_{k i}\right|+\left|a_{k j}\right|$ from classical matrix theory. So, the equality is valid.

2nd Case : Let $f_{k}\left(e_{\ell}\right) \notin f\left(e_{\ell}\right)$ for all $\ell \in N^{+}$. Then, from Definition 13

$$
\tilde{r}=\tilde{0} \quad \text { and } \quad \tilde{s}=\tilde{0}
$$

So that,

$$
|\tilde{r}+\tilde{s}|=|\tilde{0}+\tilde{0}|=\left|a_{k i}+a_{k j}\right|
$$

and

$$
|\tilde{r}|+|\tilde{s}|=|\tilde{0}|+|\tilde{0}|=\left|a_{k i}\right|+\left|a_{k j}\right|
$$

So, the equality is handed.

3rd case : Let $f_{k}\left(e_{\ell}\right) \in f\left(e_{i}\right)$ and $f_{k}\left(e_{\ell}\right) \notin f\left(e_{j}\right)$ for all $\ell \in N^{+}$. Then, $\tilde{r}=a_{k i}=1$ and $\tilde{s}=a_{k j}=\tilde{0}$. Using these, we get the below equalities,

$$
|\tilde{r}+\tilde{s}|=|\tilde{r}+\tilde{0}|=|\tilde{r}|=\left|a_{k i}\right|
$$

and

$$
|\tilde{r}|+|\tilde{s}|=\left|a_{k i}\right|+|\tilde{0}|=\left|a_{k i}\right|
$$

Finally, we have the equality.

4th case : Let $f_{k}\left(e_{\ell}\right) \notin f\left(e_{i}\right)$ and $f_{k}\left(e_{\ell}\right) \in f\left(e_{j}\right)$ for all $\ell \in N^{+}$. In this case, we can say that, $\tilde{r}=a_{k i}=\tilde{0}$ and $\tilde{s}=a_{k j}$. From this standpoint, it is obvious that we have similar equality. Then,

$$
|\tilde{r}+\tilde{s}|=|\tilde{r}|+|\tilde{s}|
$$

is valid

Let $\left\{r_{k}\right\}$ and $\left\{s_{k}\right\}$ be squences of soft real sets. Then,

$$
\sqrt[p]{\sum_{k=1}^{n}\left|r_{k}+s_{k}\right|^{p}} \leq \sqrt[p]{\sum_{k=1}^{n}\left|r_{k}\right|^{p}}+\sqrt[p]{\sum_{k=1}^{n}\left|s_{k}\right|^{p}}
$$

for all $k \in N$ and $p 1$. 1st case : Let $f_{k}\left(e_{\ell}\right) \in f\left(e_{\ell}\right)$ for all $\ell \in$ $N^{+}$. In this case, from the Definition 13

$$
\tilde{r}=a_{k i} \quad \text { and } \quad \tilde{s}=a_{k j}
$$

for all $k \in N$. Then for $p 1$,

$$
\sqrt[p]{\sum_{k=1}^{n}\left|r_{k}+s_{k}\right|^{p}}=\sqrt[p]{\sum_{k=1}^{n}\left|a_{k i}+a_{k j}\right|^{p}}
$$

and

$$
\sqrt[p]{\sum_{k=1}^{n}\left|r_{k}\right|^{p}}+\sqrt[p]{\sum_{k=1}^{n}\left|s_{k}\right|^{p}}=\sqrt[p]{\sum_{k=1}^{n}\left|a_{k i}\right|^{p}}+\sqrt[p]{\sum_{k=1}^{n}\left|a_{k j}\right|^{p}}
$$

From Minkowski inequality, the proof is handed.

In the other cases : Let $f_{k}\left(e_{\ell}\right) \notin f\left(e_{i}\right)$. Then, from the Definition 13 we get $\tilde{r}=a_{k i}=\tilde{0}$ and $\tilde{s}=a_{k j}=\tilde{0}$. It is indefinite because the radicand is zero radicand is zero in every each situation.

$$
|\tilde{r}-\tilde{s}||\tilde{r}-\tilde{t}|+|\tilde{t}-\tilde{s}|
$$

for all $\tilde{r}, \tilde{s}, \tilde{t} \in \tilde{R}(E)$

1st case : Let $f_{k}\left(e_{\ell}\right) \in f\left(e_{\ell}\right)$ for all $\ell \in N^{+}$. Then, from the Definition 13 .

$$
\tilde{r}=a_{k i} \quad, \quad \tilde{s}=a_{k j} \quad \text { and } \quad \tilde{t}=a_{k s}
$$

And then,

$$
|\tilde{r}-\tilde{s}|=\left|a_{k i}-a_{k j}\right|
$$

and

$$
\begin{aligned}
& |\tilde{r}-\tilde{t}|=\left|a_{k i}-a_{k s}\right|, \\
& |\tilde{t}-\tilde{s}|=\left|a_{k s}-a_{k j}\right| .
\end{aligned}
$$

From triangle inequality, we get the proof.

2nd case $:$ Let $f_{k}\left(e_{\ell}\right) \notin f\left(e_{\ell}\right)$ for $\forall \ell \in N^{+}$. In this case $\tilde{r}=a_{k i}=$ $\tilde{0}, \tilde{s}=a_{k j}=\tilde{0}, \tilde{t}=a_{k s}=\tilde{0}$. The equality is satisfied similarly in the 1 st case.

3rd case : Let $f_{k}\left(e_{\ell}\right) \in f\left(e_{i}\right), f_{k}\left(e_{\ell}\right) \notin f\left(e_{j}\right)$ and $f_{k}\left(e_{\ell}\right) \notin$ $f\left(e_{s}\right)$. Then,

$\tilde{r}=a_{k i}, \tilde{s}=a_{k j}=\tilde{0}, \tilde{t}=a_{k s}=\tilde{0}$.

Since,

$$
|\tilde{r}-\tilde{s}|=\left|a_{k i}-a_{k j}\right|=\left|a_{k i}\right|
$$


and

$$
\begin{gathered}
|\tilde{r}-\tilde{t}|=\left|a_{k i}-a_{k s}\right|=\left|a_{k i}\right|, \\
|\tilde{t}-\tilde{s}|=|\tilde{0}-\tilde{0}|=|\tilde{0}|
\end{gathered}
$$

the equalities are satisfied.

4th case : Let $f_{k}\left(e_{\ell}\right) \in f\left(e_{i}\right), f_{k}\left(e_{\ell}\right) \in f\left(e_{j}\right)$ and $f_{k}\left(e_{\ell}\right) \notin$ $f\left(e_{s}\right)$. Then, we get

$$
\tilde{r}=a_{k i}, \tilde{s}=a_{k j} \quad \text { and } \quad \tilde{t}=a_{k s}=\tilde{0}
$$

So, the following equalities are handed:

$$
|\tilde{r}-\tilde{s}|=\left|a_{k i}-a_{k j}\right|
$$

and

$$
\begin{aligned}
& |\tilde{r}-\tilde{t}|=\left|a_{k i}-a_{k s}\right|=\left|a_{k i}\right|, \\
& |\tilde{t}-\tilde{s}|=\left|a_{k s}-a_{k j}\right|=\left|a_{k j}\right| .
\end{aligned}
$$

The soft inequality is satisfied.

$\underline{\text { 5th case }}$ : Let $f_{k}\left(e_{\ell}\right) \in f\left(e_{i}\right), f_{k}\left(e_{\ell}\right) \notin f\left(e_{j}\right)$ and $f_{k}\left(e_{\ell}\right) \in$ $f\left(e_{s}\right)$. Then,

$$
\tilde{r}=a_{k i}, \tilde{s}=a_{k j}=\tilde{0} \quad \text { and } \quad \tilde{t}=a_{k s}
$$

From these equalities we have the following:

$$
|\tilde{r}-\tilde{s}|=\left|a_{k i}-a_{k j}\right|=\left|a_{k i}\right|
$$

and

$$
\begin{gathered}
|\tilde{r}-\tilde{t}|=\left|a_{k i}-a_{k s}\right|, \\
|\tilde{t}-\tilde{s}|=\left|a_{k s}-a_{k j}\right|=\left|a_{k s}\right|
\end{gathered}
$$

It is handed.

6th case : Let $f_{k}\left(e_{\ell}\right) \notin f\left(e_{i}\right), f_{k}\left(e_{\ell}\right) \in f\left(e_{j}\right)$ and $f_{k}\left(e_{\ell}\right) \notin$ $f\left(e_{s}\right)$. Then,

$$
\begin{gathered}
\tilde{r}=a_{k i}=\tilde{0}, \tilde{s}=a_{k j} \quad \text { and } \quad \tilde{t}=a_{k s}=\tilde{0} \\
|\tilde{r}-\tilde{s}|=\left|a_{k i}-a_{k j}\right|=\left|a_{k j}\right|
\end{gathered}
$$

and

$$
\begin{gathered}
|\tilde{r}-\tilde{t}|=\left|a_{k i}-a_{k s}\right|=|\tilde{0}-\tilde{0}|=|\tilde{0}|, \\
|\tilde{t}-\tilde{s}|=\left|a_{k s}-a_{k j}\right|=\left|a_{k j}\right|
\end{gathered}
$$

So, the equality is maintained.

7th case : Let $f_{k}\left(e_{\ell}\right) \notin f\left(e_{i}\right), f_{k}\left(e_{\ell}\right) \notin f\left(e_{j}\right)$ and $f_{k}\left(e_{\ell}\right) \in$ $f\left(e_{s}\right)$. Then,

$$
\tilde{r}=a_{k i}=\tilde{0}, \tilde{s}=a_{k j}=\tilde{0} \text { and } \tilde{t}=a_{k s}
$$

We get

$$
|\tilde{r}-\tilde{s}|=\left|a_{k i}-a_{k j}\right|=|\tilde{0}-\tilde{0}|=|\tilde{0}|
$$

and

$$
\begin{aligned}
& |\tilde{r}-\tilde{t}|=\left|a_{k i}-a_{k s}\right|=\left|a_{k s}\right|, \\
& |\tilde{t}-\tilde{s}|=\left|a_{k s}-a_{k j}\right|=\left|a_{k s}\right|
\end{aligned}
$$

equalities.

8th case : Let $f_{k}\left(e_{\ell}\right) \in f\left(e_{i}\right), f_{k}\left(e_{\ell}\right) \notin f\left(e_{j}\right)$ and $f_{k}\left(e_{\ell}\right) \notin$ $f\left(e_{s}\right)$. Then,

$$
\tilde{r}=a_{k i}, \tilde{s}=a_{k j}=\tilde{0} \quad \text { and } \quad \tilde{t}=a_{k s}=\tilde{0} .
$$

So, the following equalities are handed:

$$
|\tilde{r}-\tilde{s}|=\left|a_{k i}-a_{k j}\right|=\left|a_{k i}\right|
$$

and

$$
\begin{gathered}
|\tilde{r}-\tilde{t}|=\left|a_{k i}-a_{k s}\right|=\left|a_{k i}\right|, \\
|\tilde{t}-\tilde{s}|=\left|a_{k s}-a_{k j}\right|=|\tilde{0}-\tilde{0}|=|\tilde{0}|
\end{gathered}
$$

So, the equality is maintained.

\section{CONCLUSION}

In this work, the notion of soft matrix of soft points is introduced and its applications are presented. The main objective of this work is observe detailed examination about soft point by soft matrix method which changes with each parameter that takes place in a soft set. The scope of this approach is exhibited by storing all soft points in a systematic and unified way. The presented conceptual matrix is applied to several types of inequalities in classical set theory that are obtained as soft equalities in soft set theory and give a relation between soft set theory and classical set theory.

\section{REFERENCES}

[1] H. Aktas and N. Cağman, Soft sets and soft groups, Inform. Sci. 177 (2007), 2726-2735.

[2] M.I. Ali, F. Feng, X. Liu, W.K. Min and M. Shabir, On some new operations in soft set theory, Comput. Math. Appl. 57 (2009), 1547-1553.

[3] A. Aygŭnoğlu, H. Aygŭn, Some notes on soft topological spaces, Neural Comp. Appl. (2011), 521-011-0722-3.

[4] K.V. Babitha and J.J. Sunil, Soft set relations and functions, Comput. Math. Appl., 607 (2010), no. 7, 1840-1849.

[5] N. Cağman and S. Enginoğlu, Soft matrix theory and its decision making, Comput. Math. Appl. 59 (2010), 3308-3314.

[6] N. Cağman and S. Enginoğlu, Soft set theory and uni-int decision making, Eur. J. Oper. Res. 207 (2010), 848-855.

[7] N. Çağman, Contributions to the Theory of Soft Sets, Journal of New Result in Science, 4 (2014) 33-41.

[8] S. Das and S.K. Samanta, Soft Real Sets, Soft Real Numbers and Their Properties, J. Fuzzy Math. 20 (3) (2012) 551-576.

[9] S. Das and S.K. Samanta, Soft Metric Spaces, Annals of Fuzzy Mathematics and Informatics, 6(1) (2013) 77-94.

[10] N. TridivJyoti, S. D. Kumar, Fuzzy Soft Sets from a New Perspective Int. J. Latest Trends Computing, Vol-2 No 3 ( 2011) , pp.439-450.

[11] N. TridivJyoti, S. D. Kumar, M. Bora, On Fuzzy Soft Matrix Theory, International Journal of Mathematical Archive ISSN 2229-5046, 3(2)v(2012),pp.491-500.

[12] J. Kim, A. Baartmans. Determinant Theory for Fuzzy Matrices, Fuzzy Sets and Systems, 29(1989), pp.349-356.

[13] J. Kim, Determinant theory for Fuzzy and Boolean Matices, Congressus Numerantium Utilitus Mathematica Pub ,(1978), pp.273-276.

[14] K. Kim and F. Roush, Generalized fuzzy matrices, Fuzzy Sets and Systems, 4 (1980), pp.293-315.

[15] F. Feng, X.Y. Liu, V. Leoreanu-Fotea, Y.B. Jun, Soft sets and soft rough sets, Inform. Sci. 181 (2011), no. 6, 1125-1137.

[16] W. Gau, L, D. J. Buehrer, Vague sets, IEEE Transactions on Systems, Man, and Cybernetics, 23, 610-614, 1993. 
[17] P.K. Maji, A.R. Roy and R. Biswas, An application of soft sets in a decision making problem, Comput. Math. Appl. 44 (2002), 1077-1083.

[18] P. Majumdar and S. K. Samanta, On soft mappings, Comput. Math. Appl. 60 (2010) 2666-2672.

[19] D. Molodtsov, Soft set theory-first results, Comput. Math. Appl. 37, (1999) 19-31.

[20] D. Molodtsov, The Theory of Soft Sets (in Russian). URSS Publishers, Moscow, (2004).

[21] D.A. Molodtsov, V. Yu. Leonov and D. V. Kovkov, Soft sets technique and its application, Nechetkie Sistemy i Myagkie Vychisleniya 1 (2006), no. 1, 8-39.

[22] S. Mondal and M. Pal, Soft matrices, African Journal of Mathematics and Computer Science Research Vol. 4(13), pp. 379-388, (2011).

[23] C. Moore, Recursion theory on the reals and continuous-time computation, Theoretical Computer Science, 162 (1), 2344, (1996).

[24] Z. Pawlak, Rough sets, International Journal of Parallel Programming 11 (5), 341356, (1982).

[25] G. Senel, Soft Metric Spaces, Gaziosmanpasa University Graduate School of Natural and Applied Sciences Department of Mathematics Ph.D. Thesis, (2013), 92.

[26] G. Senel, A Comparative Research on the Definition of Soft Point, IJCA, accepted.

[27] M. G. Thomason, Convergence of powers of a fuzzy matrix, J. Math Anal. Appl. 57 (1977), pp.476-480.

[28] I. Zorlutuna, M. Akdağ, W.K. Min, S. Atmaca, Remarks on Soft Topological Spaces, Annals of Fuzzy Mathematics and Informatics, 3 (2) (2012), 171-185. 